\title{
The Effect of Absence of the Corpus Callosum on the Position of the Hippocampus and on the Formation of Probst's Bundle
}

\author{
KENNETH R. MAGEE AND ROBERT N. OLSON 1 \\ Kresge Neurological Research Laboratory, University of Michigan
}

Complete or partial agenesis of the corpus callosum results in an alteration in anatomical development of the midline structures of the cerebral hemisphere. The influence of such maldevelopment on the position of the hippocampus and its connections will be considered in this study. In addition, agenesis of the corpus callosum may allow structures existing in normal brains to become more evident because they are not masked by the abundant fiber distribution of the corpus callosum. Various other cerebral anomalies may often be seen in agenesis of the corpus callosum but they will not be stressed in this study for good reviews of the general subject are available (Baker and Graves, '33; Carpenter and Druckemiller, '53; Kirschbaum, '47; Bunts and Chaffee, '44; and Marburg, '49).

\section{PERTINENT LITERATURE}

The embryologic and phylogenetic development of the midline anatomy of the human brain will first be reviewed. In a study of marsupials, Tilney ('39) concluded, ". . . in spite of its extreme primitiveness it (the marsupial) follows, stage for stage, the fundamental principles which underlie the growth of the brain in all mammals." Tilney noted a precallosal stage in all the mammals (including man) which he examined, that eventually develop a corpus callosum.

In the marsupial, the hippocampal formation extends from the temporal pole to the olfactory peduncle and forms the medial margin of the cortex (Ariëns Kappers, Huber and Crosby, '36, pp. 1412). This midline arc of hippocampus has the same cortical makeup and general consistency throughout its extent. Along its inner margin runs a longitudinal bundle of nerve fibers, the fimbria. EIliot Smith ('10) stressed that the position of the hippocampus as the medial edge of the cerebral cortex is an important feature of the brain in the ancestors of mammals and is probably a fundamental feature in the development of all vertebrates. The dorsal commissure of the marsupial contains only fibers of hippocampal origin (fig. 1). In the bat, Nycotophilus, which represents a transition from the acallosal to the callosal brain, the anterior part of the dorsal commissure becomes invaded by pallial commissural fibers thus forming an anterior extension of the dorsal commissure (Smith 1897a). These pallial decussating fibers do not develop to so great an extent as in higher animals but begin to influence the position of the hippocampus. As in these lower forms, studies of higher mammals have demonstrated that the fibers which eventually form the corpus callosum are first seen as a bundle known as the tapetum covering the neocortical inner wall of the archicortex (Tilney, '39). When the fibers course medialward, they are pushed against the anterior side of the alveus layer of archicortex and then invade the anterior part of the dorsal commissure matrix or commissural bed of Elliot Smith (1897a).

The firsi parts of the corpus callosum to develop are the corpus, the genu, and the rostrum as these fibers are first seen in this region in front of the foramen of Monro and the anterior commissure. The increasing number of fibers causes the corpus callosum to increase in bulk and in length mainly posteriorly but there is also some forward extension. The backward extension of the corpus callosum is

\footnotetext{
1 Student fellow, National Institute of Neurological Diseases and Blindness.
} 


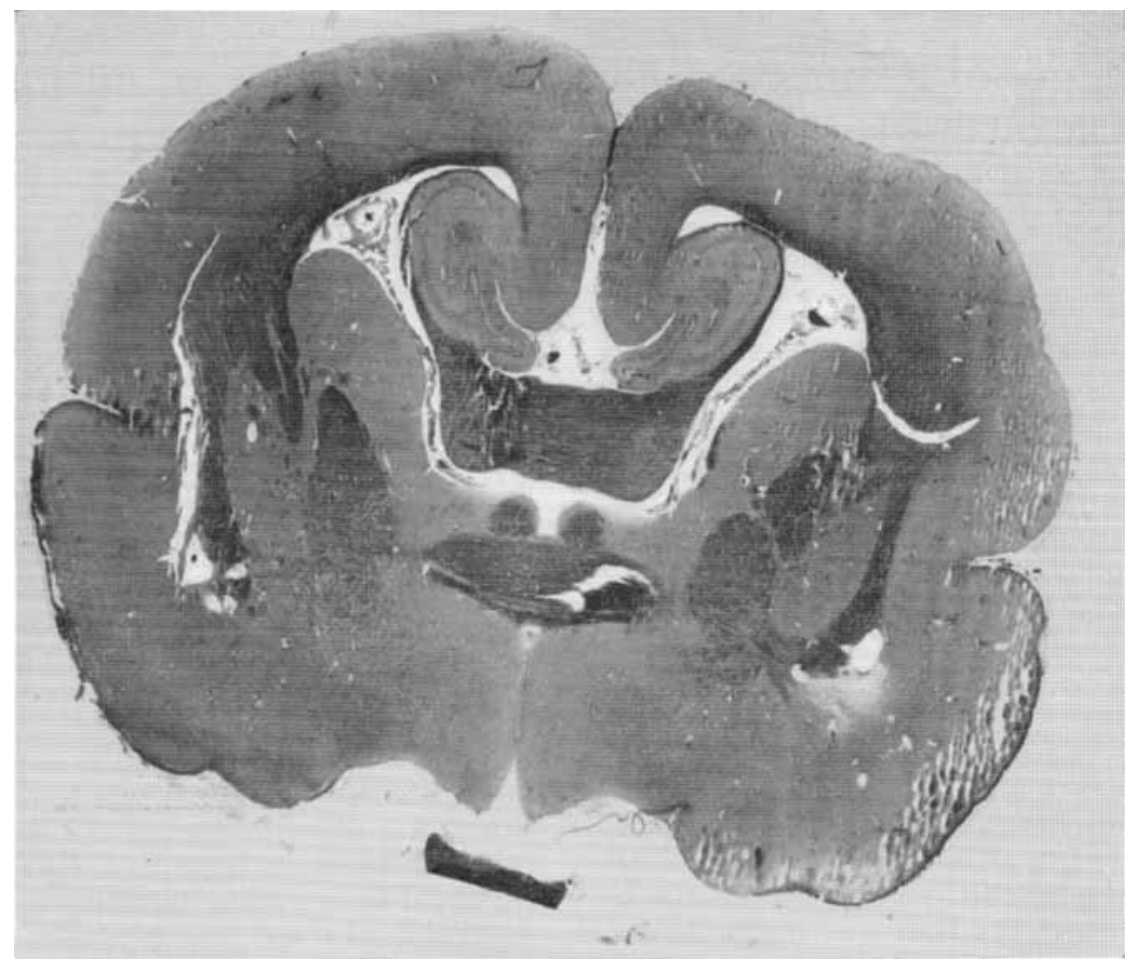

Fig. 1 Illustrating the position of the hippocampus on the medial surface of the hemisphere and the hippocampal commissure of the marsupial. Didelphis virginiana.

not accompanied by a corresponding expansion of the psalterium, which therefore becomes greatly thinned at its junction with the splenium. The posterior expansion of the corpus callosum, interposed between hippocampal formation above and hippocampal commissure below, forms the splenium (Smith, 1897a and '10). Related to the increased development of interhemispheral communication in primates, the corpus callosum is enlarged and elongated, primarily in the callosal trunk. The distance between genu and splenium is disproportionately longer than in lower mammals. The splenium is therefore carried caudad in such a way as to bear with it the hippocampal commissure and, at the same time, cause an elongation of the longitudinal fibers of the fimbria (Tilney, '39).

This caudal extension of the corpus callosum and development of the neopallial regions of the hemisphere carries with it the growing structures of the archicortex located on its dorsal surface (Tilney, '39). The hippocampal formation is therefore rolled back, down and out into the temporal pole, into close relation with the cortex of the piriform lobe (Ariëns Kappers, Huber and Crosby, '36, pp. 1413). A midline hippocampal division persists, however, forming the induseum griseum and the white stria of Lancisi. Thus, even in adult forms of higher primates, the hippocampus maintains the full arc from olfactory peduncle to temporal horn. This supracallosal hippocampus, however, is apparently retarded in development by the introduction of the corpus callosum for it does not grow or advance in cellular structure and remains very small in comparison to the hippocampus associated with the temporal horn.

The corpus callosum also influences the development of the fornix, as has been mentioned. The fornix becomes elongated with the pushing backward and downward of the hippocampus by the corpus callosum 
(Ariëns Kappers, Huber and Crosby, '36, pp. 1428). Although most fibers of the fornix are ventral to the corpus callosum, some fibers of the fornix (fimbria) system are dorsal to the corpus callosum ( $\mathrm{E}$. Smith, 1896, 1897a and b). Some of the fascicles from the medial stria of Lancisi, cross through the corpus callosum to enter the septum pellucidum where they join in the distribution of the subcallosal fornix fibers. Some fibers from the hippocampal flexure (that part of the hippocampus arching over the caudal part of the splenium ) course around the splenium before they perforate the corpus callosum. These fibers which perforate the corpus callosum to reach the fornix are called the superior fornix. It is now believed that some of these fibers may also arise from the gyrus cinguli or possibly neighboring cortical areas (Valenstein and Nauta, '59). There are also fibers which pass around the genu of the corpus callosum to reach the septum, some of which arise from the medial striae Lancisii and become a part of the precommissural fornix (Ariëns Kappers, Huber and Crosby, '36, pp. 1430).

As with the longitudinal fibers which make up the fornix systems (the fascicles of the striae of Lancisi, etc.), the major association bundles, which connect different lobes of the same hemisphere, are in close anatomical relationship with the corpus callosum. The fibers which make up these bundles vary in number and kind (short or long association fibers) at different parts of the bundle. Longitudinal association fibers exist which are not condensed into the bundles but run among the corpus callosal fibers, being perpendicular to them. With the absence of such a large structure as the corpus callosum, it is easily seen how the longitudinal fibers of the fornix longus, the fornix superior, and the association fibers of the hemispheres may come into a close relationship to form a bundle.

Considering the ways in which the development of the corpus callosum affects the position of the hippocampus and fornix, and the manner in which development of this large neopallial commissure can obscure the finer longitudinal fiber systems, it would seem quite probable that in agenesis of the corpus callosum:

(1) longitudinal association fibers and bundles, some of which are not usually distinguished, become more distinct;

(2) the location of the hippocampus and its connections is altered;

(3) early interruption or destruction of the commissural bed can be the cause of true agenesis (Marburg, 49).

The following material is presented with these observations in mind. In addition, the composition of the so-called Probst's bundle (longitudinal callosal bundle) will be noted and discussed later. This bundle is apparent in many cases of agenesis of the corpus callosum and appears as a primarily longitudinal fiber system. Its exact nature has been the subject of much disagreement.

\section{DISCUSSION OF MATERIAL}

Study 1: A 20-year-old woman had a psychosis and jerking movements of the extremities. A diagnosis of Huntington's chorea was considered but not proven. Birth and developmental history and the cause of death were not available.

Examination of the brain disclosed complete agenesis of the corpus callosum. The anterior commissure was present as was the fornix which, however, was separated throughout its full extent. Olfactory tracts were present. The septum pellucidum was absent. The gyrus cinguli was not present, although the regions of cortex which usually make up this gyrus may have been represented by portions of the convolutional pattern. Sulci radiated from what in a normal brain would have been the callosal sulcus. The parieto-occipital sulci did not meet the calcarine fissures.

The hippocampal formation was of particular interest and had not taken its normal adult position (as in the adult) but remained in a more medial and dorsal position, lying next to the medial wall of the lateral ventricle. Figure 2 shows the hippocampus in this position, superior to the inferior colliculus. Some of the fibers of Probst's bundle apparently turned downward to the hippocampus but they were not of sufficient number to account for more 


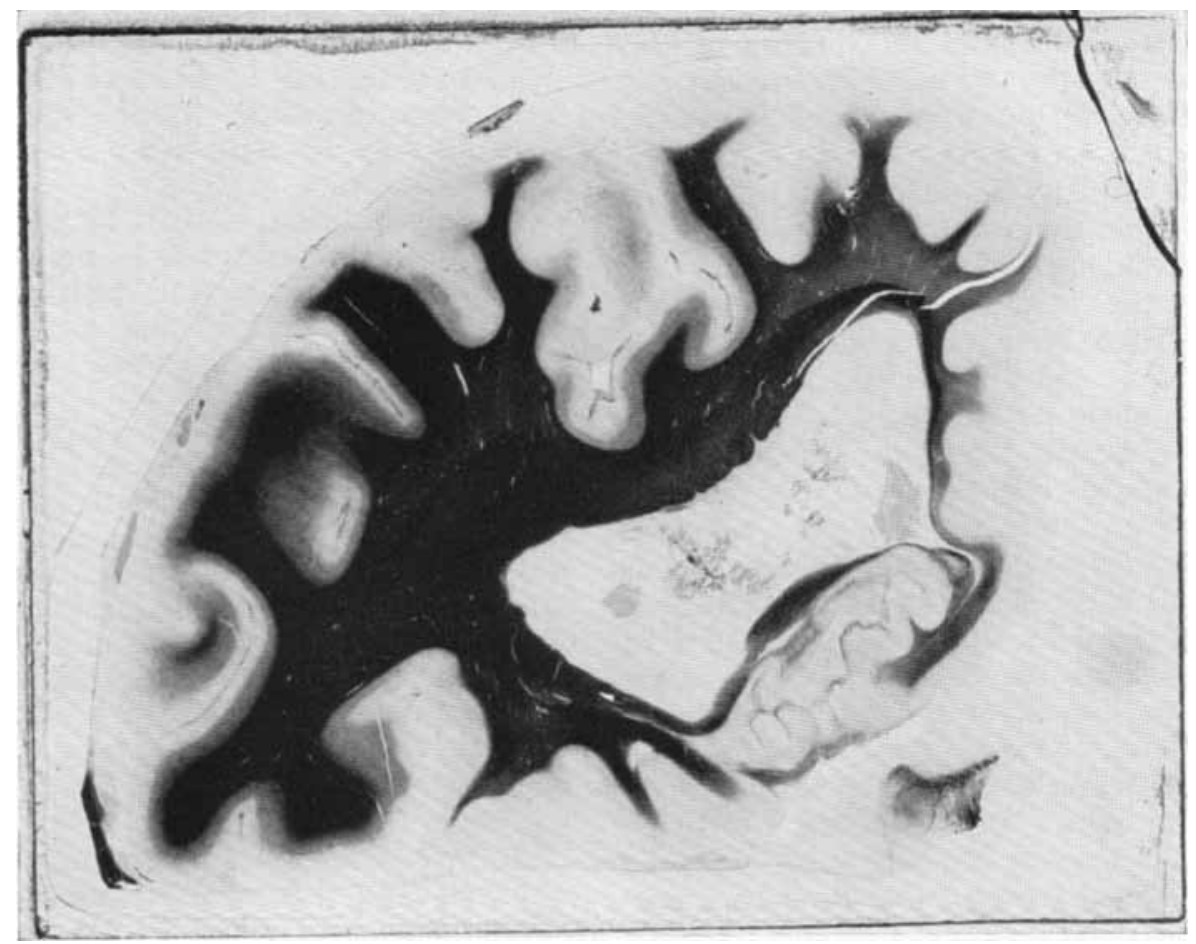

Fig. 2 Note the raised position of the hippocampus and its position superior to the inferior colliculus.

than $20 \%$ of the bundle. It is possible that a larger number of fibers from this bundle entered the hippocampus and may have turned into it rostral to the section, for serial sections of the brain were not available.

Probst's bundle extended as a protruding mass along the whole length of the lateral ventricle at the junction of the medial and the dorsal portions of the wall of the ventricle. At the level of the anterior nucleus of the thalamus, it was of greatest size and most complex structure (fig. 3 ). The fornix appeared as a tapering extension from the ventral part of the bundle and followed it along its whole protrusion into the ventricle. Fibers passed not only longitudinally but also obliquely. These oblique fibers coursed from the most ventral part of the medial cortex to the formix, therefore passing between the longitudinal fibers. Fibers arising from cortex or passing to it from the bundle first appeared to form an arch along the upper part of the bundle, which circumscribed the longitudinal fi- bers into a more compact structure. Seen in each frontal plane, some of these arching fibers ran throughout the entire extent of the bundle to reach the fornix. These "perforating" fibers thus assert their similarity to the perforating fibers of the corpus callosum.

The Probst's bundle at levels through the posterior horn of the lateral ventricle had demonstrable connections with the occipital cortex. There was also a strong continuity of the bundle with the fimbria of the abnormally placed hippocampal formation. In other words it was a combined fornix and association bundle.

Study 2: This study was made on post mortem material of the brain of a white child, who died at age two. A second cousin was feebleminded but otherwise there was no family history of neurologic disease. Retarded physical and mental development were evident. She could not sit up and had moderately hyperactive reflexes but no paralysis. The eyes were suggestive of mongolism but other stigmata of this 


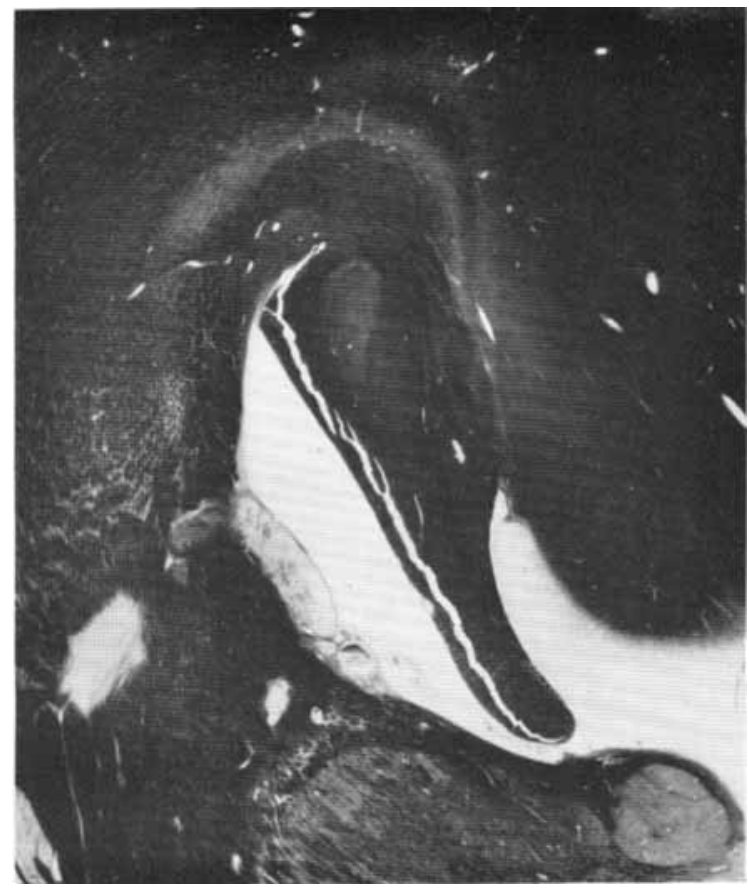

Fig. 3 Illustrating Probst's bundle at the level of the thalamus.

disorder were not evident. A diagnosis of microcephaly was made. She died of pneumonia.

Anatomical study showed fusion of the frontal lobes in the anterior portion with slight symmetrical cortical atrophy. The corpus callosum was absent. In the absence of the corpus callosum, there was a persistence of gray matter in the form of a convolution following the usual contour of what would be considered the indusium griseum over the corpus callosum in a normal brain (fig. 4).

Small hippocampi were also found in their adult location in the temporal lobes. The fimbriae were distorted although identifiable. Probst's bundle was not evident on either side. The entire brain was reduced in size. Additional findings included overall decrease in size of the cerebrum and cerebellum.

Study 3: This is a study of the brain of a man who died at 38 years of age. He had been hospitalized for 6 years before his death with a diagnosis of mental deficiency, high imbecile grade. No information was available about birth history, early development, or family history. Four years before death, he developed an embryonal carcinoma (monocellular type) of the right testicle. This eventually resulted in death due to massive retroperitoneal lymph node metastases with ureteral obstruction and uremia.

The brain was completely fused in both frontal lobes with heterotopic gray masses scattered throughout, but mainly on either side of the midline. The gray near the midline resembled hippocampal tissue and was in an area corresponding to the position of the induseum griseum when the corpus callosum is present (fig. 5). The rostrum, the genu, and the body of the corpus callosum were absent but the splenium was present. The ventricular system was not divided as the septum pellucidum was absent. A $Y$ shaped single cavity was formed. The hippocampi were bilaterally high and inverted (figs. $6 a$ and b).

Study 4: A 53-year-old female was "simple and backward" in school. Mental symptoms of withdrawal, hallucinations, and aggressive behavior developed in adult life. Two cousins were also mentally re- 


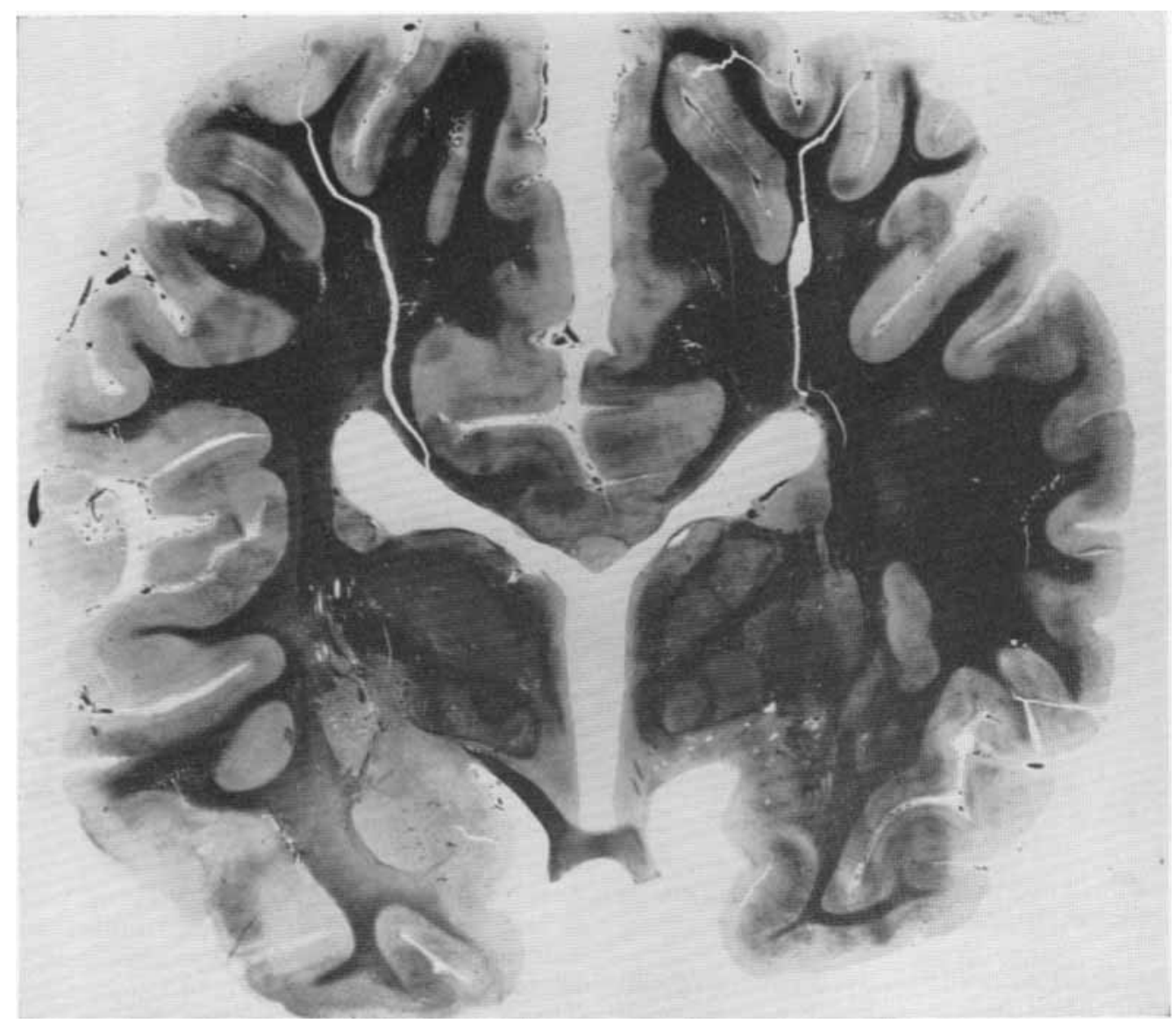

Fig. 4 Note small convolution corresponding to the area where the induseum griseum would be found in a callosal brain.

tarded. Examinations, shortly before death, disclosed organic mental deficiency with a superimposed psychotic reaction. The neurologic examination was otherwise normal. The patient died of chronic myocarditis.

Post mortem examination of the brain disclosed an agenesis of the corpus callosum. The hippocampi were in normal location but were small. Probst's bundle was present. Fibers were seen entering it from the cingulate gyrus. The connections of Probst's bundle were the same in study 1 (fig. 7).

Study 5: A 7-year-old child, who had had a normal birth and no family history of neurologic disease, showed early retarded mental and physical development. At the age of 7 , she could say only a few words. She could never walk. Neurologic examination disclosed, in addition to severe mental retardation, spasticity of all extremities with contractures in her joints. She died of bronchopneumonia. On routine autopsy, a congenital absence of the gall bladder was found.

The convolutional pattern was abnormal; macrogyria were present in the frontal and the parietal lobes and microgyria in the occipital lobe. The parieto-occipital fissure did not meet the calcarine fissure. The sulci in the parietal and the occipital regions radiated from the callosal sulcus. The cingulate gyrus was not present although there were longitudinal gyri in the frontal lobe which were interrupted by short shallow sulci. The occipital lobe was larger than normal (fig, 8). There was no 

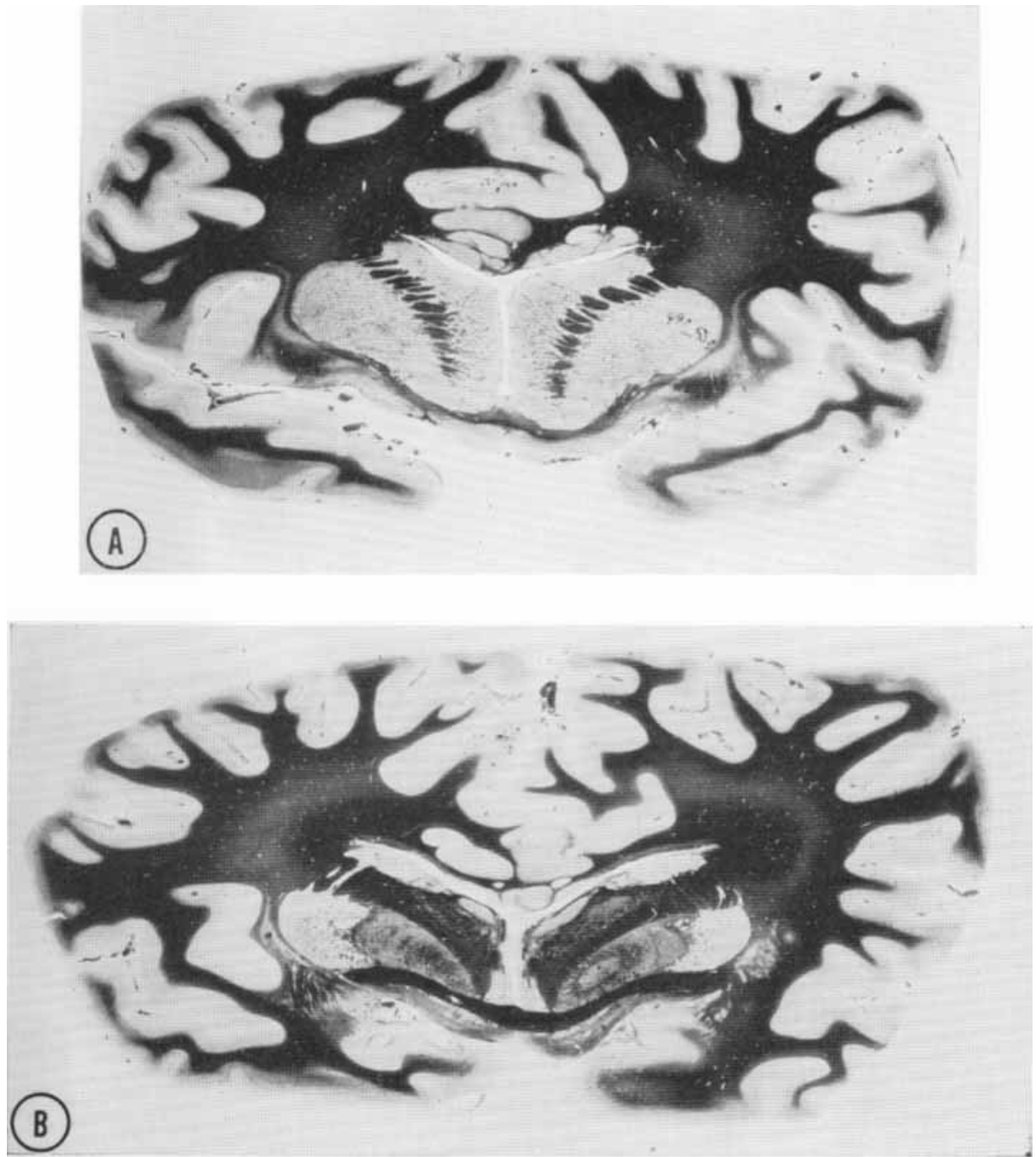

Fig. 5a and $b$ Note gray matter dorsal to the lateral ventricle in the area where the induseum griseum would be expected in a callosal brain.

anterior commissure or septum pellucidum. The fornix was present but divided.

A Probst's bundle was present and extended along the dorsomedial portion of the lateral ventricle. It also extended along the medial margin of the ventricle forming with the fornix, which tapered from its ventral surface, an arch around the lower part of the lowest convolutions of the medial cortex. Fibers coursing between the Probst's bundle and the cingulate gyrus were abundant. Connections were also present between the Probst's bundle and other parts of the frontal cortex. Longitudinal fibers in this bundle were not so great as in the other cases but perforating fibers were abundant.

At the lateral border of the roof of the ventricle and extending down the lateral surface was a bundle of longitudinally running fibers. This bundle, evident in this brain, but not in all of the others, was composed of association fibers and represented the fibers normally seen in the superior 

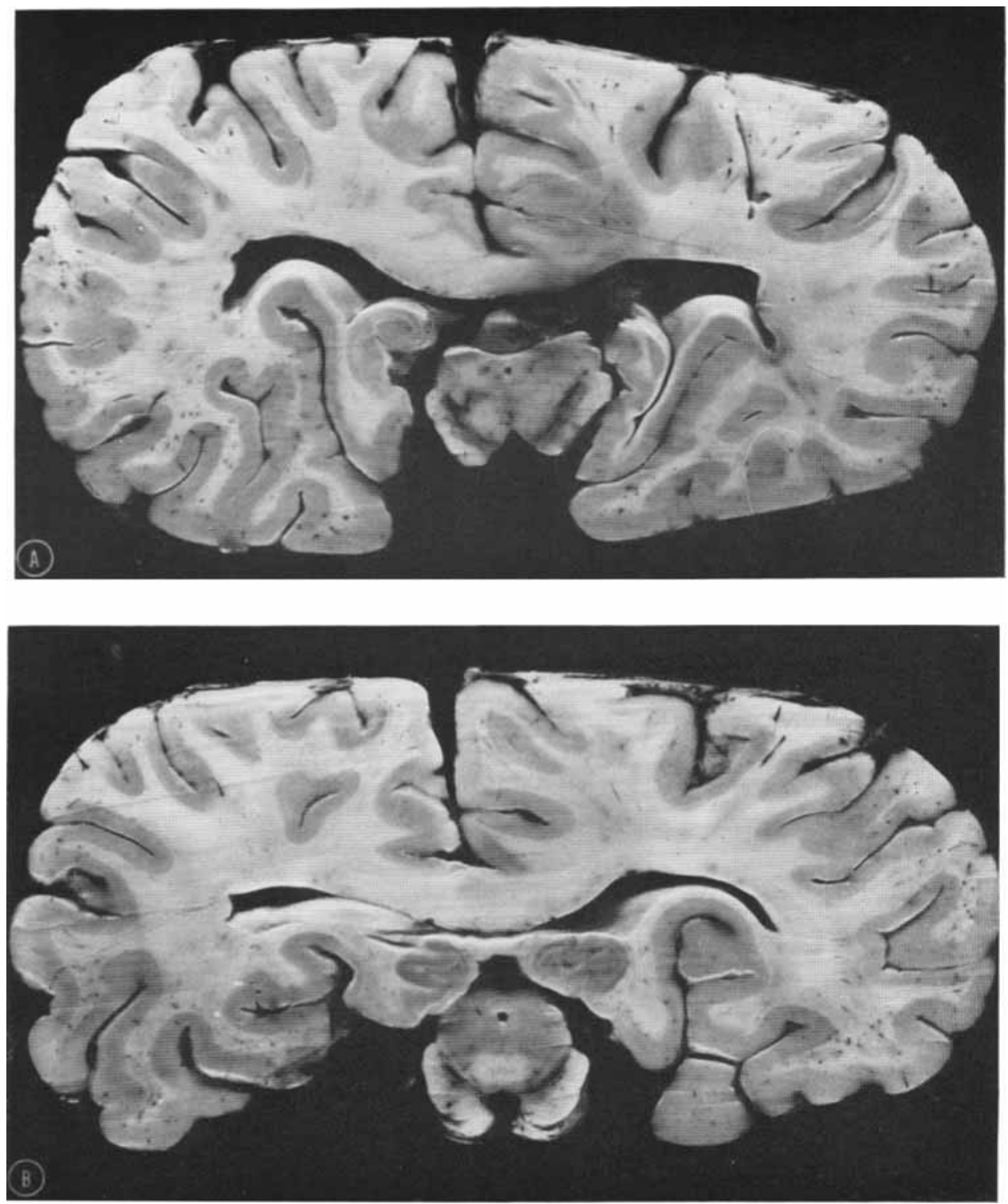

Fig. $6 \mathrm{a}$ and $\mathrm{b}$ Note the high inverted position of the hippocampi.

occipito-frontal fasciculus. The cingulum and the stratum subcallosum could not be identified.

The findings in this brain indicate that the composition of the Probst's bundle may vary, depending on how close the fibers group together in the absence of the corpus callosum. Here the superior occipitofrontal fasciculus was not incorporated into the Probst's bundle but was a separate recognizable fiber fasciculus close to it.

\section{DISCUSSION}

The brains in studies 1,2 and 3 illustrate that, in agenesis of the corpus callosum, abnormal accumulations of gray matter were present in an area corresponding to the location of the induseum griseum in 


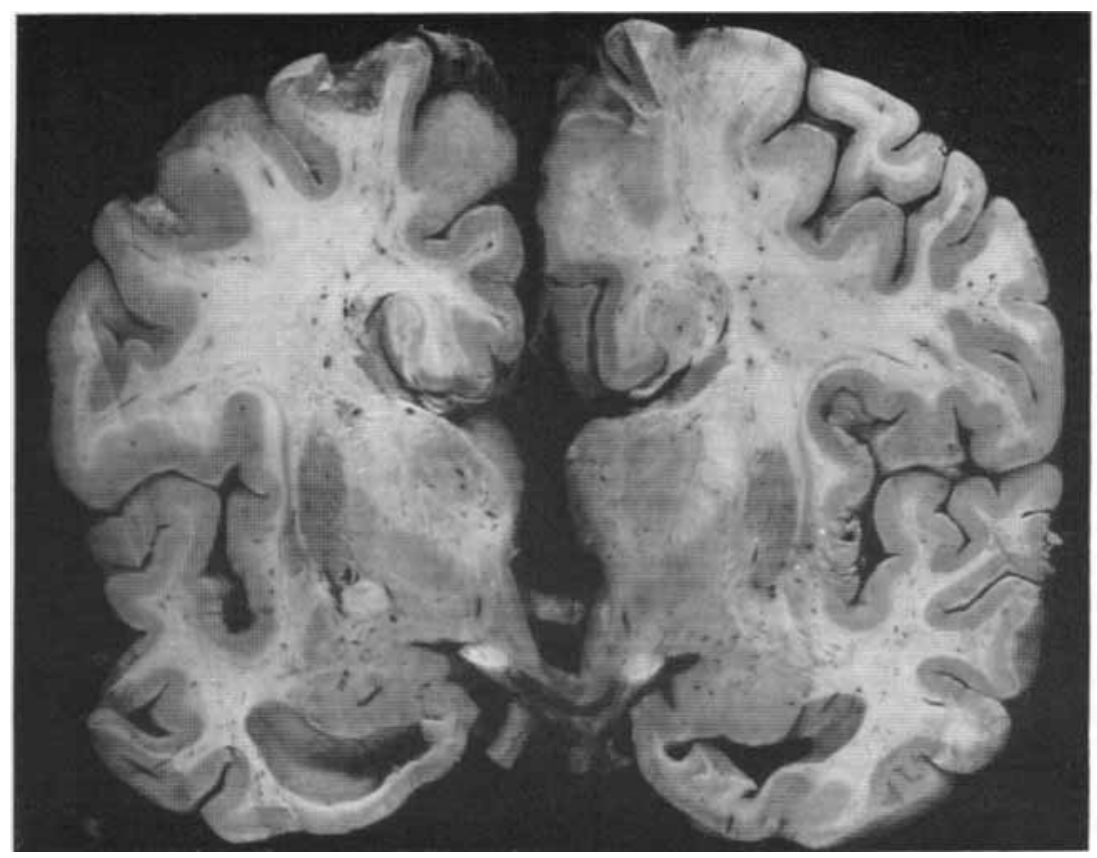

Fig. 7 Note agenesis of the corpus callosum with a well-formed Probst's bundle in the dorsolateral wall of the lateral ventricle. It is best seen on the right. The convolution on the left in the same area as the bundle on the right resembles the hippocampus but its connections could not be traced adequately.

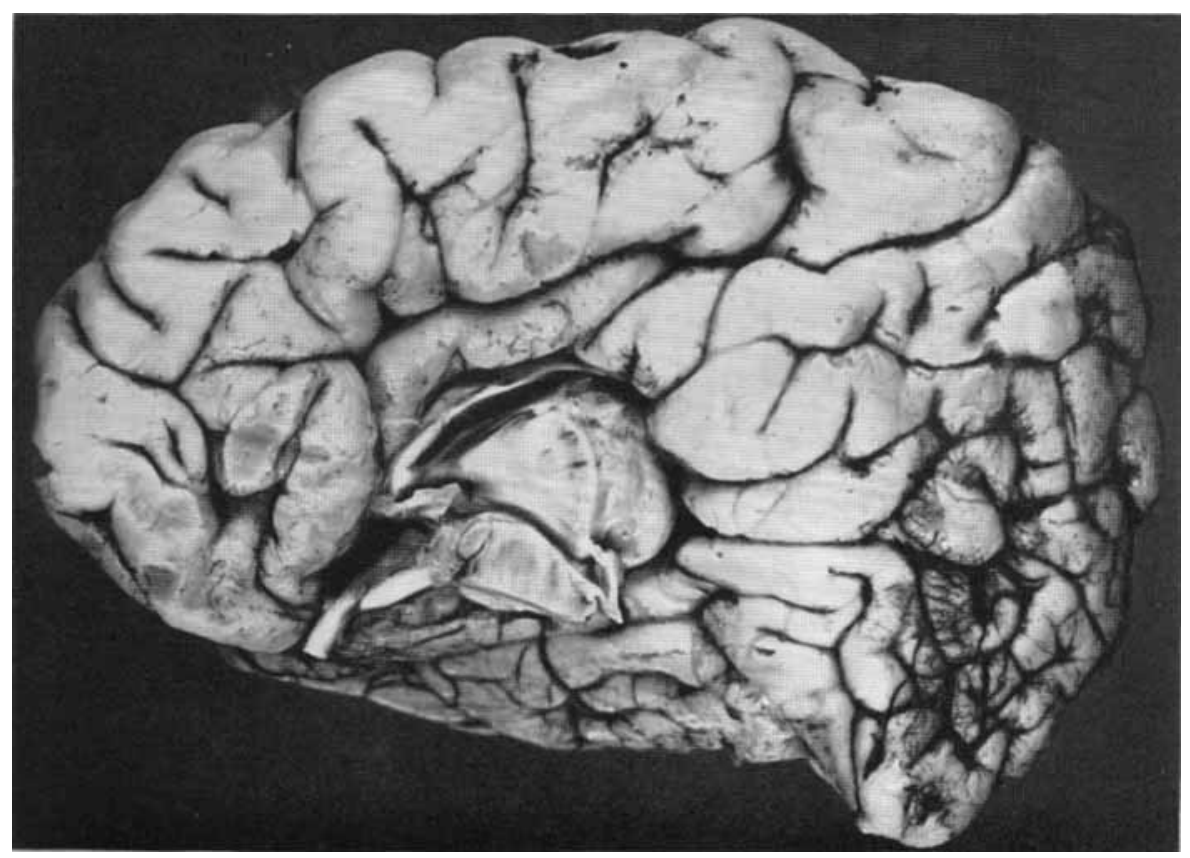

Fig. 8 Note agenesis of the corpus callosum and the distorted convolutional pattern. 
the normal brain. Such remnants of gray matter were quite likely hippocampal tissue which has not been carried into its normal adult position by the developing corpus callosum. Added evidence that such aberrant tissue is hippocampal in origin is afforded by the first brain where the clearly identifiable hippocampus can be seen rising, from its normal position in the temporal horn of the ventricle, to a location dorsal and medial to the wall of the lateral ventricle (fig. 2).

It is unfortunate that the material available was not accompanied by sufficient information to allow judgment as to whether the cerebral malformation was of genetic origin or due to birth trauma or a similar secondary process.

From the location of the hippocampal tissue, one might hope to determine whether the absence of the corpus callosum was a true agenesis or whether this commissure had secondarily degenerated after once forming. In the latter situation, one would expect the hippocampus to retain its final position at the temporal pole of the ventricle. If the corpus callosum had never formed or the commissural plate had been destroyed early in development, varying degrees of hippocampal tissue might be expected in the more primitive position on the medial hemispheric surface. Bruce (1889) and O. Marburg ('49) have given criteria for determining when the defect has occurred. Marburg stated that true agenesis of the corpus callosum occurs during the first 14 days of life, but developmental defects of the corpus callosum occur during the second half of fetal life and a complete agenesis does not occur.

Although absence of the corpus callosum has been shown to alter the position of the hippocampus as described above, it is equally true that in some cases of absence of the corpus callosum where there is no obvious evidence to suggest a degenerative process, the hippocampus finds its way to the adult position. Even in the cases described in this paper varying amounts of hippocampal tissue were observed in the normal position. This would indicate that additional factors besides the development of the corpus callosum and expansion of the neocortex may contribute to the adult position of the hippocampus.

Other authors have noted hippocampal tissue near the medial surface of the hemisphere in acallosal brains. Shryock, Barnard and Knighton ('40) demonstrated a large aberrant mass of cortical tissue taken across the central sulcus of an acallosal brain that clearly resembled the hippocampus. Kuhlenbeck and Globus ('36) presented a somewhat different anomaly - arhinencephaly with extreme eversion of the end-brain in which the corpus callosum was rudimentary and a clearly formed supracallosal hippocampus was evident. Kirschbaum (47) described heterotopic gray matter forming a bridge between the frontal olfactory and the hippocampal system. He commented that this case had certain features in common with the arrangement of olfactory cortex in lower mammals but he tended to doubt that congenital anomalies represented reversions to the ancestral condition. Lichtenstein and Maloney ('54) also described two cases where failure of migration of the hippocampus were evident in the anomalous brains.

The origin of Probst's bundle (longitudinal callosal bundle, Balkenlangsbündel of Probst ('01)) has been in dispute. According to some observers the bundle is a normal structure which becomes more distinctly visible with the lack of callosal $f-$ bers. This implies that the bundle is composed of association pathways between various lobes of the hemisphere which appear unrelated when the corpus callosum is well developed. Other investigators have felt that the bundle consists of aberrant callosal fibers which, instead of crossing to the other hemisphere, assume a sagittal direction. The most detailed account of the bundle in the English language is that of deLange ('25). After a careful review of the subject, delange presented a brain with partial agenesis of the corpus callosum in which, instead of a splenium, there was a "pseudosplenium," fibers of which radiated to the cortex in a manner similar to that of a normal splenium and which had a position comparable to a longitudinally running Probst's bundle. She concluded, therefore, that the Probst's bundle contained aberrant, uncrossed fibers of the 
corpus callosum. Recently, Akert, Puletti, and Erickson ('54), suggested that a band of heterotopic white matter in the postcentral gyrus of an acallosal brain represented misdirected callosal fibers. They also suggest that Probst's bundle is of the same origin. Cameron ('07) and DouglasCrawford ('06) described acallosal brains which have a Probst's bundle and persistance of gray matter on the medial surface of the hemisphere from the uncus to the olfactory region. They suggested that these structures are representatives of the limbic lobe and that the longitudinal fibers of Probst's bundle are related to the fornix system, the longitudinal stria and connections of the cortex with the limbic lobe. Cameron also suggested that some of these fibers are a condensation of the normal long longitudinal intrahemispheric connections.

It is difficult to accept the conclusion that fibers that normally cross and provide interhemispheral communication assume instead a long aberrant longitudinal course and end indiscriminately in various regions on the same side of the brain. It seems more probable that association fibers between different regions of the same hemisphere which are masked or separated from each other in the normal brain by the fibers of the corpus callosum, when the latter structure does not exist, come into closer relationship to each other. Thus a tract (Probst's bundle), not recognizable in the normal brain, is formed. That many of these fibers arise from areas similar to the fibers of the corpus callosum is not disturbing for this most likely occurs in the normal brain. It is suggested, therefore, that Probst's bundle is composed of at least the following systems: (1) Septal-hippocampal, hippocampal-septal fibers and possible other fornix components; (2) the superior fornix of Elliot Smith; (3) association fibers between various parts of other cortical areas.

The material presented showed that at least some of the fibers in Probst's bundle are projection fibers of the hippocampus or septum pellucidum. It is obvious that the bundle, when present as a distinct entity, is not composed of just one group of fibers but of fibers of various types. This correlates with the hypothesis in the preceding paragraph.

\section{ACKNOWLEDGMENT}

We wish to acknowledge with deep gratitude the guidance and assistance of Dr. Elizabeth C. Crosby throughout this study.

\section{LITERATURE CITED}

Akert, K., F. Puletti and T. C. Erickson 1954 Abnormalities of the cerebral cortex associated with agenesis of corpus callosum and focal cortical seizure. Trans. Am. Neur. Assoc., 79: 151-153.

Ariëns Kappers, C. U., G. C. Huber and E. C. Crosby 1936 The comparative anatomy of the nervous system of vertebrates, including man. The MacMillan Co., New York.

Baker, R. C., and G. O. Graves 1933 Partial agenesis of the corpus callosum. Arch. Neur. Psychiat., 29; 1054-1065.

Bruce, A. 1889 On the absence of the corpus callosum in the human brain, with the description of a new case. Brain, 12: 171-190.

Bunts, A. T., and J. S. Chaffee 1944 Agenesis of the corpus callosum with possible porencephaly: review of the literature and a report of a case. Arch. Neur. Psychiat., 51: 35-53.

Cameron, J. L. 1907 A brain with complete absence of the corpus callosum. J. Anat. Physiol., 41: 293-301.

Carpenter, M. B., and W. H. Druckemiller 1953 Agenesis of the corpus callosum diagnosed during life. A.M.A. Arch. Neur. Psychiat., 69: 305322.

DeLange, C. 1925 On brains with total and partial lack of the corpus callosum and on the nature of the logitudinal callosal bundle. $J$. Nerv. Ment. Dis., 62: 449-476.

Douglas-Crawford, D. 1905 A case of absence of the corpus callosum. J. Anat. Physiol., 40: $57-62$.

Kirschbaum, W. R. 1947 Agenesis of the corpus callosum and associated malformations. J. Neuropath. Exp. Neur., 6: 78-94.

Kuhlenbeck, H., and J. H. Globus 1936 Arhinencephaly with extreme eversion of the endbrain. Arch. Neur. Psychiat., 36; 58-74.

Lichtenstein, B. W., and J. E. Maloney 1954 Malformation of the forebrain with comments on the so-called dorsal cyst. The corpus callosum, and the hippocampal structures. J. Neuropath. Exp. Neur., 13: 117-128.

Marburg, O. 1949 So-called agenesia of the corpus callosum (Callosal defect): Anterior cerebral dysraphism. Arch. Neur. Psychiat, 61: 297-312.

Probst, M. 1901 Ueber den Bau des vollständig balkenlosen Grosshirns sowie über Mikrogyrie and Heterotopie der grauen Substanz. Arch. Psychiat., 34: 709-786.

Shryock, E. H., J. F. Barnard and R. S. Knighton 1940 Agenesis of the corpus callosum associated with porencephaly: report of a case. Bull. Los Angeles Neur. Soc., 5: 146-163. 
Smith, G. Elliot 1896 The "fornix superior." J. Anat. Physiol., 31: 80-94.

$1897 \mathrm{a}$ The origin of the corpus callosum: a comparative study of the hippocampal region of the cerebrum of Marsupialia and certain Cheiroptera. Tr. Linnean Soc. Lond. Zoology 2nd. Ser, 7: 47-69.

$1897 \mathrm{~b}$ The relation of the fornix to the margin of the cerebral cortex. J. Anat. Physiol., $32 ; 23-58$.
1910 Some problems relating to the evolution of the brain. Lancet., 1: 1-6.

Tilney, F. 1939 The hippocampus and its relations to the corpus callosum. J. Nerv. Ment. Dis., 89: 433-513.

Valenstein, E. S., and W. J. H. Nauta 1959 A comparison of the distribution of the fornix system in the rat, guinea pig, cat, and monkey. J. Comp. Neur., 113: 337-363. 